\title{
Difference in the Pharmacokinetics and Hepatic Metabolism of Antidiabetic Drugs in Zucker Diabetic Fatty and Sprague-Dawley Rats ${ }^{\mathbb{}}$
}

\author{
Xin Zhou, Luc R. A. Rougée, David W. Bedwell, Jeff W. Cramer, Michael A. Mohutsky, \\ Nathan A. Calvert, Richard D. Moulton, Kenneth C. Cassidy, Nathan P. Yumibe, Lisa A. Adams, \\ and Kenneth J. Ruterbories
}

Drug Disposition (X.Z., L.R.A.R., D.W.B., J.W.C., M.A.M., N.A.C., R.D.M., K.C.C., N.P.Y., K.J.R.) and Tailoring Therapeutics (L.A.A.), Lilly Research Laboratories, Indianapolis, Indiana

Received March 22, 2016; accepted May 20, 2016

\begin{abstract}
The Zucker diabetic fatty (ZDF) rat, an inbred strain of obese Zucker fatty rat, develops early onset of insulin resistance and displays hyperglycemia and hyperlipidemia. The phenotypic changes resemble human type 2 diabetes associated with obesity and therefore the strain is used as a pharmacological model for type 2 diabetes. The aim of the current study was to compare the pharmacokinetics and hepatic metabolism in male ZDF and Sprague-Dawley (SD) rats of five antidiabetic drugs that are known to be cleared via various mechanisms. Among the drugs examined, metformin, cleared through renal excretion, and rosiglitazone, metabolized by hepatic cytochrome P450 2C, did not exhibit differences in the plasma clearance in ZDF and SD rats. In contrast, glibenclamide, metabolized by hepatic CYP3A, canagliflozin, metabolized mainly by UDP-glucuronosyltransferases (UGT), and
\end{abstract}

troglitazone, metabolized by sulfotransferase and UGT, exhibited significantly lower plasma clearance in ZDF than in SD rats after a single intravenous administration. To elucidate the mechanisms for the difference in the drug clearance, studies were performed to characterize the activity of hepatic drug-metabolizing enzymes using liver $\mathbf{S 9}$ fractions from the two strains. The results revealed that the activity for CYP3A and UGT was decreased in ZDF rats using the probe substrates, and decreased unbound intrinsic clearance in vitro for glibenclamide, canagliflozin, and troglitazone was consistent with lower plasma clearance in vivo. The difference in pharmacokinetics of these two strains may complicate pharmacokinetic/ pharmacodynamic correlations, given that ZDF is used as a pharmacological model, and SD rat as the pharmacokinetics and toxicology strain.

\section{Introduction}

Zucker diabetic fatty (ZDF) rats are inbred from the Zucker fatty rat, an obese Zucker strain that carries a spontaneous mutation of the leptin receptor gene, and males display persistent hyperglycemia (Peterson et al., 1990; Truett et al., 1991; Phillips et al., 1996). Female ZDF rats display the phenotype of obesity and insulin resistance with a level comparable to male ZDF rats but rarely exhibit spontaneous hyperglycemia (Clark et al., 1983; Peterson et al., 1990). In contrast, male ZDF rats develop obesity and insulin resistance as early as 7-weeks old and progressively develop hyperglycemia and hyperlipidemia with aging. At 14 weeks of age, male ZDF rats display higher body weight and body mass index as well as significantly higher levels of plasma insulin, glucose, and fasting glucose compared with their lean littermates (Kava et al., 1990; Aleixandre de Artinano and Miguel Castro, 2009). The hyperglycemia is accompanied by impaired pancreatic $\beta$-cell function,

dx.doi.org/10.1124/dmd.116.070623.

SThis article has supplemental material available at dmd.aspetjournals.org. loss of $\beta$-cell mass, and impaired response of liver and extrahepatic tissues to the actions of insulin and glucose (Shiota and Printz, 2012). These features, including the development of obesity- and hyperglycemia-related complications, are common between male ZDF rats and humans with obesity-associated type 2 diabetes. Therefore, ZDF rats are widely used as a pharmacology model for studying obese type 2 diabetes in drug discovery (Shiota and Printz, 2012). However, the pharmacokinetics (PK) and toxicology studies in drug discovery are typically performed in the Sprague-Dawley (SD) rat.

Pharmacokinetics and metabolic differences between the original obese Zucker strain and SD rats have previously been reported. Phenobarbital PK were found to be different between obese Zucker and lean Zucker or SD rats (Brouwer et al., 1984), whereas altered expression and/or activity of hepatic drug metabolism enzymes cytochrome P450 2E1, CYP4A2, glutathione- $S$-transferase(s), and UDP-glucuronosyltransferases (UGT) were demonstrated in obese Zucker rats compared with their lean littermates (Litterst, 1980; Chaudhary et al., 1993; Enriquez et al., 1999). Recent work has shown that hepatic mRNA expression levels of Phase I and Phase II enzymes,

ABBREVIATIONS: $C L_{\text {int }}$, intrinsic clearance; canagliflozin, (1S)-1,5-anhydro-1-[3-[[5-(4-fluorophenyl)-2-thienyl]methyl]-4-methylphenyl]-D-glucitol, free base; 4-MU, 4-methylumbelliferone; 4-MUG, 4-methylumbelliferyl- $\beta$-D-glucuronide; HPLC, high-performance liquid chromatography; LC-MS/MS, liquid chromatography tandem-mass spectrometry; PAPS, adenosine 3'-phosphate 5'-phosphosulfate; PD, pharmacodynamic; P450, cytochrome P450; PK, pharmacokinetics; PPAR, peroxisome proliferator-activated receptor; SD, Sprague-Dawley; SULT, sulfotransferase; $t_{1 / 2}$, terminal half-life; UDPGA, uridine $5^{\prime}$-diphospho-glucuronic acid; UGT, UDP-glucuronosyltransferases; $V d_{\mathrm{ss}}$, volume of distribution at steady state; ZDF, Zucker diabetic fatty. 
including CYP3A1, CYP2C11, UGT1A1, UGT1A6, UGT2B1, GSTA2, and quinone reductase, as well as xenobiotic transporters Mrp2, Mrp3, and Oatp2, were downregulated in obese Zucker compared with SD rats (Kim et al., 2004). The altered expression of the UGT enzymes, coupled with decreased UGT activity measured in liver microsomes, was the attributed cause of a significant reduction in clearance of two peroxisome proliferator-activated receptor (PPAR) antagonists in vivo, which are primarily cleared via UGT-mediated acyl glucuronidation followed by biliary excretion of acyl glucuronide metabolite (Kim et al., 2004). Although diabetic phenotypes are well characterized in ZDF rats (Shiota and Printz, 2012), little is known about the expression and activity of hepatic drug-metabolizing enzymes or drug PK in this strain.

In the present study, the PK for the antidiabetic drugs metformin (Sterne, 1959), rosiglitazone (Lehmann et al., 1995), glibenclamide (also known as glyburide, Bhattia et al., 1970), canagliflozin (Nomura et al., 2010), and troglitazone (Fujiwara et al., 1988), which represent different clearance mechanisms (renal excretion and hepatic metabolism) and metabolic pathways (P450, UGT, and sulfotransferase), were characterized in male ZDF and SD rats after a single dose of intravenous administration. Compound-specific differences in plasma clearance between the two strains led to further characterization of the activities of hepatic metabolic enzymes CYP3A, UGTs, and sulfotransferase(s) using probe substrates testosterone (Ning et al., 2012) and midazolam (Giroux et al., 2016), 4-methylumbelliferone (Shiratani et al., 2008), and 7-hydroxycoumarin (van den Berg et al., 2013), respectively, to elucidate potential differences in the hepatic metabolism capability via these pathways. These findings should help in interpreting the comparison of PK and toxicology data gathered from SD rats with efficacy and pharmacology data performed in ZDF rats in drug discovery.

\section{Materials and Methods}

Chemicals and Reagents. Rosiglitazone free base (( \pm )-5-[[4-[2-(methyl-2pyridinylamino)ethoxy]phenyl]methyl]-2,4-thiazolidinedione), troglitazone free base ( \pm -5-[[4-[(3,4-dihydro-6-hydroxy-2,5,7,8-tetramethyl-2H-1-benzopyran-2yl)methoxy]phenyl]methyl]-2,4-thiazolidinedione), metformin hydrochloride ( $N, N$-dimethylimidodicarbonimidic diamide hydrochloride), and glibenclamide free base (1-[[p-[2-(5-chloro-o-anisamido)ethyl]phenyl]-sulfonyl]-3-cyclohexylurea) (Fig. 1) were synthesized at Lilly Research Laboratories (Indianapolis, IN). Other chemical reagents were purchased commercially: canagliflozin ((1S)-1,5-anhydro1-[3-[[5-(4-fluorophenyl)-2-thienyl]methyl]-4-methylphenyl]-D-glucitol, free base) (Selleck Chemicals, Houston, TX); midazolam, $\alpha$-hydroxymidazolam, $\alpha$-hydroxymidazolam- $\mathrm{D}_{4}, 6 \beta$-hydroxytestosterone, and $6 \beta$-hydroxytestosterone- $\mathrm{D}_{3}$ (Cerilliant, Round Rock, TX); 7-hydroxycoumarin sulfate and 7-hydroxycoumarin sulfate- $\mathrm{D}_{5}$ (Toronto Research Chemicals Inc., Toronto,
Canada); testosterone, 4-methylumbelliferone (4-MU), 4-methylumbelliferyl$\beta$-D-glucuronide (4-MUG), 7-hydroxycoumarin, alamethicin, NADPH, uridine 5 '-diphospho-glucuronic acid (UDPGA), and adenosine 3'-phosphate 5' phosphosulfate (PAPS) (Sigma-Aldrich, St. Louis, MO). Solvents (dimethylsulfoxide, methanol, acetonitrile) were of high-performance liquid chromatography (HPLC) grade (Fisher Scientific, Pittsburg, PA).

Animals. Age-matched male SD and ZDF rats were obtained from Charles River Laboratories Inc. (Wilmington, MA). Rats were 14-weeks-old, weighing $300-450 \mathrm{~g}$ for both strains, with their femoral arteries and veins surgically cannulated. After delivery to Covance (Greenfield, IN), rats were acclimated for at least 3 days prior to study initiation. Access to food and water was allowed ad libitum. All animal procedures were approved by the Institutional Animal Care and Use Committee at Covance.

In Vivo Pharmacokinetic Studies. Rosiglitazone, troglitazone, metformin hydrochloride, canagliflozin, and glibenclamide were administered intravenously via a femoral vein, at $1 \mathrm{mg} / \mathrm{kg}(1 \mathrm{ml} / \mathrm{kg})$ in $25 \mathrm{mM}$ phosphate buffer $(\mathrm{pH} 8)$ containing $10 \%$ dimethylacetamide $(\mathrm{v} / \mathrm{v}), 15 \%$ ethanol $(\mathrm{v} / \mathrm{v})$, and $30 \%$ propylene glycol $(\mathrm{v} / \mathrm{v})$. Serial blood samples were collected from the femoral artery at 0.08 , $0.25,0.5,1,2,4,8,12$, and 24 -hours postdose and treated with $\mathrm{K}_{3}$-EDTA. Plasma was obtained by centrifugation at $1600 \mathrm{~g}$ for 10 minutes, and stored at $-70^{\circ} \mathrm{C}$ until analysis by liquid chromatography tandem-mass spectrometry (LC-MS/MS).

Determination of Pharmacokinetic Parameters. Noncompartmental pharmacokinetic parameters were calculated using Watson version 7.4 (Thermo Scientific, Waltham, MA). Statistical significance of differences for pharmacokinetic parameters between two groups was examined with Student's $t$ test using Sigmaplot 12.5 (Systat Software Inc., San Jose, CA).

Metabolite Identification in Hepatocytes. Hepatocyte incubations and metabolite profiling were conducted at $\mathrm{Q}^{2}$ Solutions, a Quintiles Quest Joint Venture (Indianapolis, IN). In short, hepatocytes from male SD rats and male human donors were obtained from BioreclamationIVT (Baltimore, MD). Incubations are performed in a $\mathrm{CO}_{2}$ incubator at $37^{\circ} \mathrm{C}$ using a 24 -well plate containing 250,000 cells/well. A stock solution of compound was added to medium to give a final incubation concentration of $10 \mu \mathrm{M}$. The incubations including the media and cells were quenched with an equal volume of acetonitrile after 2 hours. Samples were centrifuged at $3500 \mathrm{~g}$ for 10 minutes and the supernatant was analyzed by liquid chromatography coupled to a diode array UV detection and mass spectrometry system consisting of the Acquity ultra-performance liquid chromatography column, Synapt G2-S mass spectrometer, and a ultra-performance liquid chromatography photodiode array detector (Waters, Milford, MA). The chromatographic separation of metabolites was achieved on a Waters 1.7- $\mu$ m Acquity BEH C18 $(100 \times 2.1 \mathrm{~mm})$ column maintained at $60^{\circ} \mathrm{C}$. The mobile phase consisted of solvent $\mathrm{A}(10 \mathrm{mM}$ ammonium bicarbonate in water) and solvent B (100\% acetonitrile). The samples were eluted, at a flow rate of $0.5 \mathrm{ml} / \mathrm{min}$ with a gradient for 5.5 minutes. The LC/MS full scan data were processed by Metabolynx (Waters, Milford, MA) to identify metabolites. Product ion spectra were acquired to confirm the metabolites. The mass spectrometer was operated in negative ion mode with an electrospray ionization source. The parameters for the chamber were: capillary $1.0 \mathrm{kV}$, sample cone $50 \mathrm{~V}$, source temperature $120^{\circ} \mathrm{C}$, desolvation $500^{\circ} \mathrm{C}$, and collision energy $25 \mathrm{~V}$.
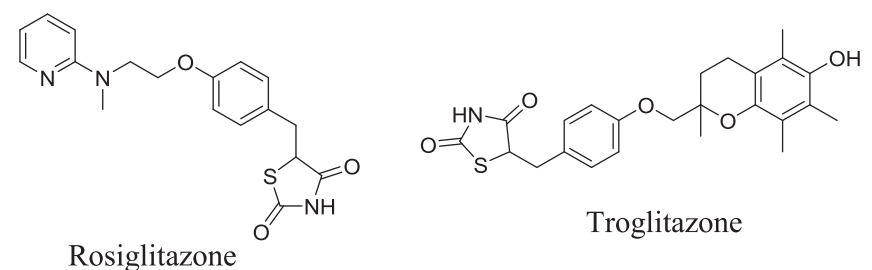

$$
\text { Metformin }
$$<smiles>COc1ccc(Cl)cc1C(=O)NCCc1ccc(S(=O)(=O)NC(=O)NC2CCCCC2)cc1</smiles>

Glibenclamide

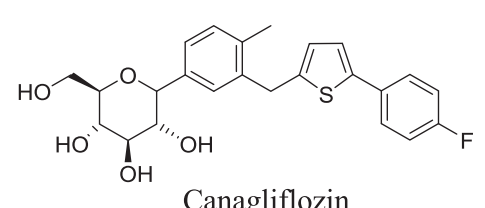

Canagliflozin
Fig. 1. Chemical structures of antidiabetic drugs in the present study. 
Determination of Enzyme Activity in Liver S9. Pooled S9 fraction from livers of 10 animals, 14- to 16-week-old male SD and ZDF rats, was obtained from Corning Gentest (Tewksbury, MA). Reactions were conducted under air in a shaking water bath at $37^{\circ} \mathrm{C}$. Substrates, buffer, and microsomes were premixed, and incubations were initiated by addition of the reaction-specific cofactor. Final concentration of carrier solvent was less than $1 \%$.

The probe substrate midazolam and testosterone for CYP3A, 4-MU for UGTs, and 7-hydroxycoumarin for sulfotransferases (SULTs) were used to assess the difference in the enzyme activity in S9 of the two strains. The rate for the formation of the metabolites $\alpha$-hydroxymidazolam, $6 \beta$-hydroxytestosterone, 4-MUG, and 7-hydroxycoumarin sulfate, respectively, was determined. Assay mixtures for midazolam and testosterone contained $100 \mathrm{mM}$ potassium phosphate buffer (pH 7.4), $30 \mu \mathrm{M}$ midazolam or $200 \mu \mathrm{M}$ testosterone, and $0.5 \mathrm{mg} / \mathrm{ml}$ liver S9 protein. Reactions were preincubated for 3 minutes at $37^{\circ} \mathrm{C}$ prior to initiation by the addition of $1 \mathrm{mM}$ NADPH and allowed to proceed for 5 minutes and 10 minutes for midazolam and testosterone. Incubations of 4-MU contained $50 \mathrm{mM}$ Tris buffer (pH 7.4) supplemented with $10 \mathrm{mM} \mathrm{MgCl} 2,10 \mu \mathrm{g} / \mathrm{ml}$ alamethicin, $100 \mu \mathrm{M}$ substrate, and $0.1 \mathrm{mg} / \mathrm{ml} \mathrm{S} 9$ protein. After preincubation, reactions were initiated by addition of $5 \mathrm{mM}$ UDPGA and allowed to proceed for 10 minutes. Assay for 7-hydroxycoumarin contained $100 \mathrm{mM}$ Tris buffer ( $\mathrm{pH}$ 7.4), $25 \mu \mathrm{M}$ substrate, and $0.4 \mathrm{mg} / \mathrm{ml} \mathrm{S9}$ protein. Cofactor PAPS $(0.1 \mathrm{mM})$ was used to initiate the reactions that were carried out at $37^{\circ} \mathrm{C}$ for 10 minutes.

At the end of each incubation time period, reaction aliquots ( $50 \mu \mathrm{l}$ for testosterone and midazolam; $10 \mu \mathrm{l}$ for 4-MU and 7-hydroxycoumarin) were quenched into $100 \mu \mathrm{l}$ or $190 \mu \mathrm{l}$, respectively, of 90:10 v/v $\left(\mathrm{MeOH} / \mathrm{H}_{2} \mathrm{O}\right)$ containing an internal standard for analysis (see Supplemental Table 1 for details). Plates were sealed with Easy Pierce $20-\mu \mathrm{m}$ foil (Thermo Fisher Scientific), vortexed for 20 seconds, and centrifuged (3500g for 10 minutes) and analyzed by LC-MS/MS.

The intrinsic clearance for parent loss of four antidiabetic drugs was determined in liver S9 fraction from SD and ZDF rats for rosiglitazone, glibenclamide, and canagliflozin in a NADPH-fortified system, canagliflozin and troglitazone in a UDPGA-fortified system, and troglitazone in a PAPSfortified system, as described above for the incubation of probe substrate except that substrate concentration was at $0.1 \mu \mathrm{M}$ with $\mathrm{S} 9$ protein at $0.5 \mathrm{mg} / \mathrm{ml}$ for all compounds except for troglitazone, which was performed at $0.25 \mathrm{mg} / \mathrm{ml}$. Aliquots $(50 \mu \mathrm{l})$ were taken at $0,2.5,5,10,15,20$, and 30 minutes of incubation for all reaction combinations except for glibenclamide $(0,2.5,5,15$, 30,60 , and 120 minutes) and canagliflozin $(0,15,30,45,60,90$, and 120 minutes) with NADPH and quenched into $100 \mu \mathrm{l}$ of 90:10 v/v $\left(\mathrm{MeOH} / \mathrm{H}_{2} \mathrm{O}\right)$ containing an internal standard for analysis (see Supplemental Table 1 for details). Plates were sealed, vortexed for 20 seconds, centrifuged ( $3500 \mathrm{~g}$ for 10 minutes), and analyzed by LC-MS/MS using standard curves prepared under identical conditions.

All incubations were conducted on three separate protein aliquots, and each reaction was performed in triplicate. Statistical significance of differences of the average of the separate protein aliquots (mean of replicate averages) between two strains was examined with unpaired two-tail Student's $t$ test.

Analytical Instrumentation and General Aspects of Bioanalysis. Study samples were analyzed by LC-MS/MS using either an AB Sciex API 6500 or AB Sciex 5500 triple quadrupole mass spectrometer (Applied Biosystems/MDS, Foster City, CA) equipped with a TurbolonSpray interface. The pumps were Shimadzu LC-10AD units with a SCL-10A controller (Kyoto, Japan), and a PAL liquid handler (CTC Analytics AG, Zwingen, Switzerland) was used as the autosampler. Chromatography was performed at ambient temperature. All compounds were chromatographically separated using a Betasil javelin C18 $2 \times 20-\mathrm{mm} 5-\mu \mathrm{m}$ HPLC column (Thermo Fisher Scientific), except for testosterone, $6 \beta$-hydroxytestosterone, midazolam, $\alpha$-hydroxymidazolam, 7-hydroxycoumarin, and 7-hydroxycoumarin sulfate, which were separated using an Ace UltraCore C18 $30 \times 2.1-\mathrm{mm} 5-\mu \mathrm{m}$, HPLC column (Advanced Chromatography Technologies Ltd., Aberdeen, UK). Analytical details for instrument, aqueous mobile phase, organic mobile phase, MS/MS transition, collision energy, and internal standard are summarized in Supplemental Table 1.

Determination of Plasma and S9 Protein Binding. Rat plasma protein binding was determined in vitro using equilibrium dialysis, as described previously (Zamek-Gliszczynski et al., 2011). Rat liver S9 protein binding was determined in vitro following the same protocol and using $0.5 \mathrm{mg} / \mathrm{ml}$ liver $\mathrm{S} 9$ protein from SD and ZDF rats for the incubation for all compounds, except troglitazone and 7-hydroxycoumarin for which protein concentrations of
0.25 and $0.4 \mathrm{mg} / \mathrm{ml}$ were used to mimic assay conditions. Statistical significance of differences between SD and ZDF was examined using a Student's $t$ test.

\section{Results}

Pharmacokinetics of Five Anti-Diabetic Drugs. Plasma clearance of the five antidiabetic drugs, intravenously administered at $1 \mathrm{mg} / \mathrm{kg}$, was compared between 14-week-old male SD and ZDF rats (Fig. 2). Body weights for male ZDF and SD rats were comparable, which is distinctive from obese Zucker rats that have been shown to have a body weight two times greater than SD rats at 14-weeks old (Kim et al., 2004). The plasma clearance obtained by dividing the dose by area under the concentration-time curve from time zero extrapolated to infinity from noncompartmental analysis indicated that elimination of rosiglitazone, canagliflozin, and glibenclamide from plasma was much lower than rat hepatic blood flow $\left(Q_{\mathrm{h}}=55 \mathrm{ml} / \mathrm{min}\right.$ per kilogram) (Davies and Morris, 1993). Elimination of metformin and troglitazone was rapid in rats, via renal secretion and hepatic metabolism, respectively (Table 1). No difference was observed for clearance of metformin and rosiglitazone between ZDF and SD rats. However, canagliflozin, glibenclamide, and troglitazone exhibited lower plasma clearance in ZDF than SD rats. The terminal half-life $\left(t_{1 / 2}\right)$ was significantly longer for canagliflozin and glibenclamide, reflecting a reduction in elimination rate constant. Although the $t_{1 / 2}$ was longer for troglitazone in ZDF rats compared with SD rats; this did not reach statistical significance. The volume of distribution at steady state $\left(V d_{\mathrm{ss}}\right)$ for ZDF rats was significantly higher for glibenclamide, lower for canagliflozin, and unchanged for troglitazone compared with SD rats, representing the combined effects in alteration of the elimination rate constant and area under the concentration-time curve from noncompartmental analysis (Table 1).

Identification of Metabolites Formed by Rat and Human Hepatocytes. Metabolite profiles were determined for canagliflozin, glibenclamide, troglitazone, and rosiglitazone, the four drugs mainly cleared by hepatic metabolism in rats and humans (Fig. 3; Table 2). A species difference was observed for canagliflozin regarding the abundance of metabolite formation. Metabolite A, the alcohol oxidation product, was the predominant metabolite in rat and also has been reported as a rodent-specific product (Mamidi et al., 2014), whereas the glucuronidation metabolite $\mathrm{B}$ was the most abundant in human (Fig. 3A; Table 2). Therefore, intrinsic clearance $\left(C L_{\text {int }}\right)$ of canagliflozin in liver S9 fraction of SD and ZDF rats was assessed in both NADPH- and UGPGA-fortified system. Multiple oxidation metabolites for glibenclamide, including an alkane hydroxylation metabolite A, cyclohexane hydroxylation metabolites B, C, D, and $\mathrm{E}$, and a $\mathrm{N}$-dealkylation metabolite $\mathrm{F}$ were detected in both rat and human hepatocytes (Fig. 3B; Table 2), suggesting P450-mediated oxidation as the major metabolic pathway for hepatic metabolism. For troglitazone, the sulfation metabolite B was identified as the most abundant metabolite formed from both rat and human hepatocytes. The oxidation metabolite $\mathrm{A}$ and glucuronidation metabolite $\mathrm{C}$, as well as multiple secondary metabolites were identified as minor metabolites (Fig. 3C; Table 2). Finally, P450-mediated metabolism represented the major metabolic pathways in hepatocytes for rosiglitazone, with $\mathrm{N}$-desmethyl product $\mathrm{B}$ identified as the major metabolite in both rat and human hepatocytes, accompanied by formation of oxidation metabolites $\mathrm{A}$ and $\mathrm{E}$, and other secondary metabolites (Fig. 3D; Table 2).

In Vitro Activity in Hepatic S9 Using Probe Substrates. The total yield of protein for S9 fraction extracted from pooled liver of 10 male rats at the age of 14- to 16-weeks was comparable between SD and ZDF rats $(60 \mathrm{mg} / \mathrm{gram}$ for ZDF versus $58 \mathrm{mg} / \mathrm{gram}$ for SD liver tissues, 
A

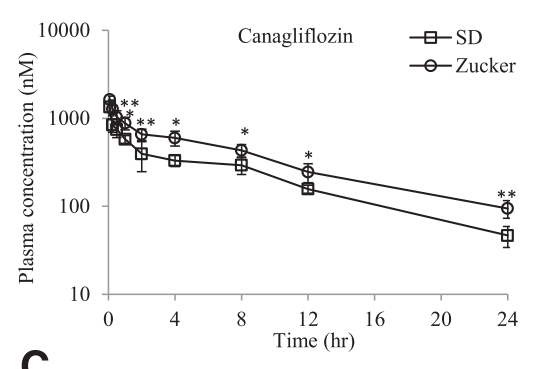

C

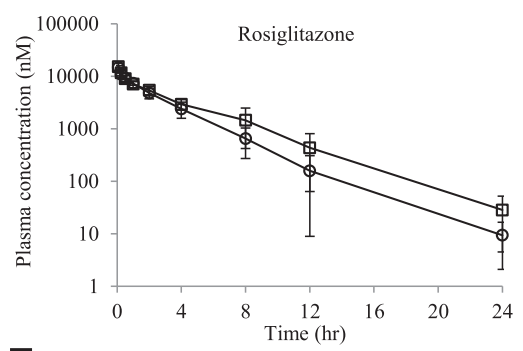

E

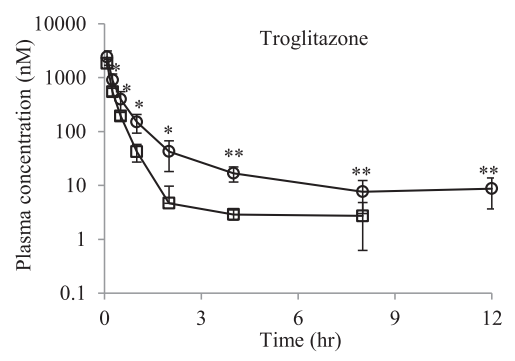

B
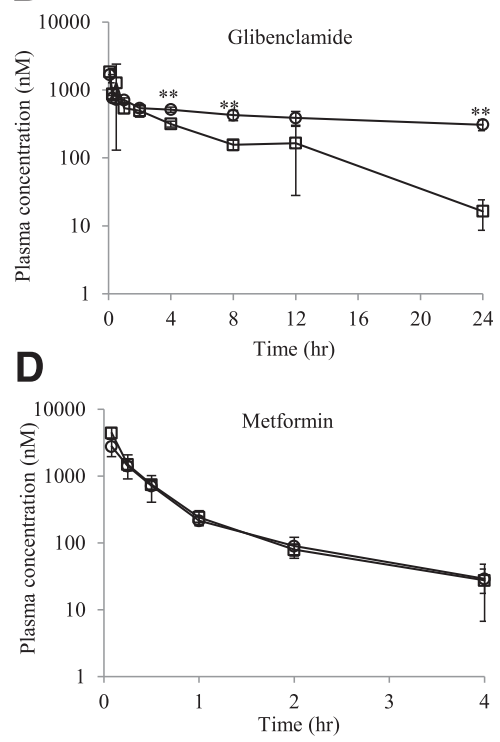

Fig. 2. Plasma concentration of anti-diabetic drugs (A) Canagliflozin, (B) Glibenclamide, (C) Rosiglitazone, (D) Metformin and (E) Troglitazone in ZDF and SD rat after a single intravenous administration at $1 \mathrm{mg} / \mathrm{kg}$. Data points represent mean \pm S.D. $(n=4)$. $* P<0.05$ and $* * P<0.01$ compared with concentration in SD rats. respectively). The rate of formation of metabolites $6 \beta$-hydroxytestosterone and 1-hydroxymidazolam for CYP3A, 4-MU glucuronide (4-MUG) for UGT, and 7-hydroxycoumarin sulfate for SULT was assessed and corrected for the unbound liver S9 fraction of each substrate (Fig. 4). The results demonstrated that the activity for CYP3A and UGT enzymes was decreased in ZDF rats (Fig. 4, A-C), whereas the activity of SULT enzymes was comparable between two strains (Fig. 4D). Substrate concentration in the assays were higher than $K_{\mathrm{m}}$ values of the probe substrates, representing a condition of enzyme activity close to $V_{\text {max }}$. This suggests that the differences in activity mainly reflect differences in the enzyme expression.

Parent Loss of Diabetic Drugs in Hepatic S9. The in vitro $C L_{\text {int }}$ calculated through parent compound depletion was examined in the presence of $0.1 \mu \mathrm{M}$ substrate, a concentration relevant to the unbound liver concentration after intravenous administration at $1 \mathrm{mg} / \mathrm{kg}$.
Incubations were conducted in a NADPH-, UDPGA-, or PAPSfortified system according to the metabolite profile obtained from rat hepatocytes. No difference in hepatic metabolism of rosiglitazone by cytochrome P450-mediated oxidation in S9 of ZDF or SD rats was observed (Fig. 5A). This is consistent with the unaltered $\mathrm{PK}$ in vivo from the two strains. Unbound $C L_{\text {int }}$ for glibenclamide was significantly lower in ZDF compared with SD rats in the presence of NADPH, also consistent with the lower clearance observed in vivo (Fig. 5B). Troglitazone depletion via both UGT glucuronidation and SULT sulfation were significantly lower in ZDF compared with SD rats (Fig. 5, C-D). Canagliflozin loss via P450 (Fig. 5E) was significantly lower in ZDF than SD rats, whereas loss through UGT glucuronidation was comparable between the two strains (Fig. 5F).

In Vitro Plasma and Liver S9 Protein Binding. The unbound plasma fraction was below quantitative limit for troglitazone,

TABLE 1

Plasma pharmacokinetic parameters for anti-diabetic drugs in SD and ZDF rats

Rats were given drugs at $1 \mathrm{mg} / \mathrm{kg}$ i.v. via femoral vein. Values were derived from plots shown in Fig. 2. Results shown represent mean \pm standard deviation from four animals.

\begin{tabular}{|c|c|c|c|c|c|c|c|c|c|c|}
\hline \multirow{2}{*}{ Parameters } & \multicolumn{2}{|c|}{ Canagliflozin } & \multicolumn{2}{|c|}{ Glibenclamide } & \multicolumn{2}{|c|}{ Rosiglitazone } & \multicolumn{2}{|c|}{ Metformin } & \multicolumn{2}{|c|}{ Troglitazone } \\
\hline & $\mathrm{SD}$ & ZDF & SD & ZDF & SD & ZDF & SD & $\mathrm{ZDF}$ & SD & ZDF \\
\hline $\operatorname{AUC}\left(\mu \mathrm{M}^{*} \mathrm{~h}\right)$ & $5.4 \pm 0.6$ & $8.6 \pm 1.6^{a}$ & $5.1 \pm 1.5$ & $10 \pm 1.5^{b}$ & $40 \pm 12$ & $32 \pm 7$ & $1.9 \pm 0.4$ & $1.5 \pm 0.2$ & $0.6 \pm 0.1$ & $1.1 \pm 0.4^{a}$ \\
\hline $\mathrm{AUC}_{\mathrm{inf}}(\mu \mathrm{M} * \mathrm{~h})$ & $5.8 \pm 0.7$ & $9.7 \pm 1.8^{a}$ & $5.3 \pm 1.6$ & $24 \pm 6.3^{b}$ & $40 \pm 12$ & $32 \pm 7$ & $1.9 \pm 0.4$ & $1.5 \pm 0.2$ & $0.6 \pm 0.1$ & $1.3 \pm 0.6^{a}$ \\
\hline $\mathrm{CL}(\mathrm{ml} / \mathrm{min}$ per $\mathrm{kg})$ & $6.5 \pm 0.8$ & $4.0 \pm 0.8^{b}$ & $6.8 \pm 1.6$ & $1.5 \pm 0.5^{b}$ & $1.2 \pm 0.3$ & $1.5 \pm 0.3$ & $71 \pm 16$ & $90 \pm 14$ & $63 \pm 10$ & $35 \pm 15^{a}$ \\
\hline$V d_{\mathrm{ss}}(1 / \mathrm{kg})$ & $3.4 \pm 0.3$ & $2.4 \pm 0.3^{b}$ & $2.4 \pm 0.2$ & $3.6 \pm 0.5^{b}$ & $0.3 \pm 0.0$ & $0.2 \pm 0.0$ & $3.4 \pm 2.3$ & $3.7 \pm 0.7$ & $1.6 \pm 0.6$ & $4.7 \pm 3.2$ \\
\hline$t_{1 / 2}(\mathrm{~h})$ & $6.2 \pm 0.6$ & $7.6 \pm 0.5^{b}$ & $4.8 \pm 0.8$ & $31 \pm 9^{b}$ & $2.9 \pm 0.2$ & $2.7 \pm 0.1$ & $2.6 \pm 3.6$ & $1.1 \pm 0.4$ & $2.0 \pm 1.2$ & $5.6 \pm 4.2$ \\
\hline
\end{tabular}

$\mathrm{AUC}$, area under the concentration-time curve; $\mathrm{AUC}_{\mathrm{inf}}, \mathrm{AUC}$ extrapolated from time zero extrapolated to infinity; $V d_{\mathrm{ss}}$, volume of distribution at steady state ${ }^{a} P<0.05$ with Student's $t$ test.

${ }^{b} P<0.01$ with Student's $t$ test. 


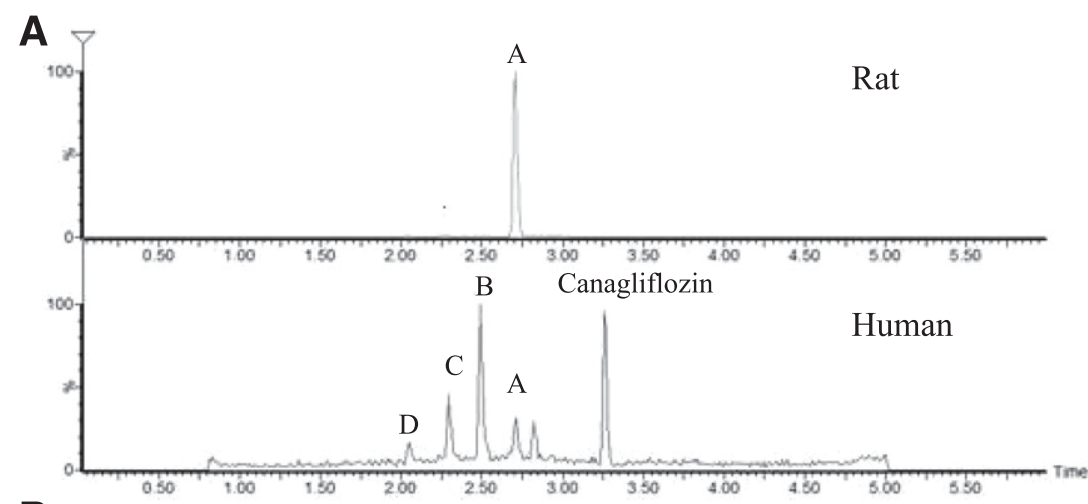

B



C



D

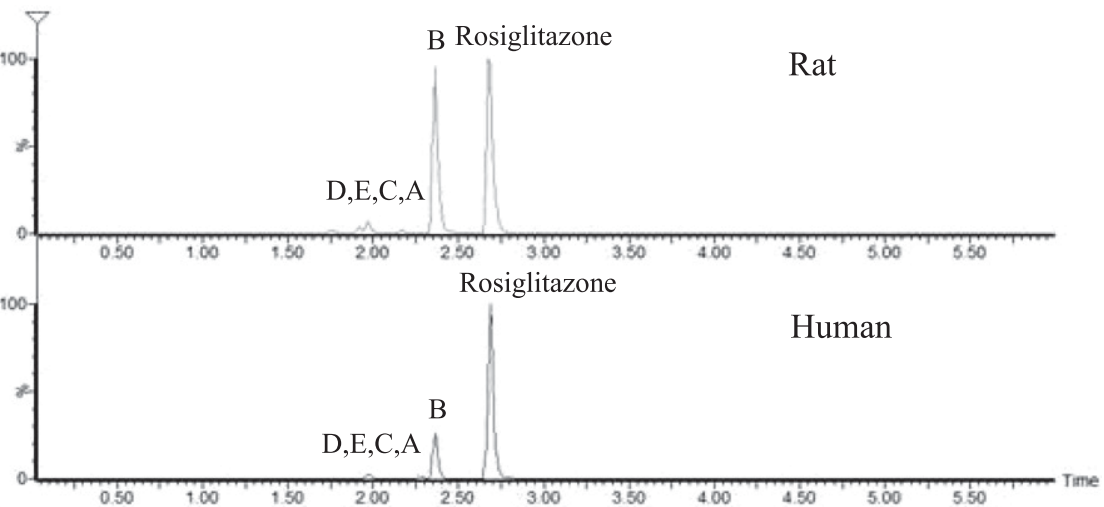

Fig. 3. Metabolite identification for antidiabetic drugs (A) Canagliflozin, (B) Glibenclamide, (C) Troglitazone and (D) Rosiglitazone in hepatocytes of SD rats. Reaction mixtures contained 250,000 hepatocytes per well and drug at $10 \mu \mathrm{M}$ concentration. Incubation and metabolite analysis were performed with a Waters LC/MS instrument as described in Materials and Methods. rosiglitazone, canagliflozin, and glibenclamide with current methods (data not shown), given that plasma protein binding is greater than $99 \%$ for the drugs examined. However, the unbound liver S9 fraction was within detection limits and was significantly higher in ZDF compared with SD rats for testosterone, midazolam, rosiglitazone, canagliflozin, and troglitazone (Table 3).

\section{Discussion}

Among the antidiabetic drugs examined in the present study, rosiglitazone and metformin showed no difference in plasma clearance between the $\mathrm{ZDF}$ and $\mathrm{SD}$ rat strains. In the case of rosiglitazone, primarily metabolized in humans by hepatic $\mathrm{CYP} 2 \mathrm{C} 8$ to form $\mathrm{N}$-demethylation and hydroxylation metabolites (Baldwin et al., 1999; 
TABLE 2

Identification of metabolites produced in rat and human hepatocytes

Reaction mixtures contained 250,000 hepatocytes per incubation and drug at $10 \mu \mathrm{M}$ concentration. Metabolite analysis was performed with a Waters LC/MS instrument, as described under Materials and Methods. Tentative metabolite identification based on MS/MS fragmentation, $m / z$, retention time, and species identified are shown.

\begin{tabular}{|c|c|c|c|c|c|}
\hline Drug & Metabolite & Tentative Metabolite Identification & $m / z$ & $\mathrm{RT}(\min )$ & Species \\
\hline \multirow[t]{5}{*}{ Canagliflozin } & & Parent & 443 & 3.26 & $\mathrm{HH}$ \\
\hline & A & $\mathrm{P}+\mathrm{O}-2 \mathrm{H}$ & 457 & 2.71 & $\mathrm{RH}, \mathrm{HH}$ \\
\hline & $\mathrm{B}$ & $\mathrm{P}+$ glucuronide & 619 & 2.49 & $\mathrm{HH}$ \\
\hline & $\mathrm{C}$ & $\mathrm{P}+\mathrm{O}+$ sulfate & 539 & 2.29 & $\mathrm{RH}, \mathrm{HH}$ \\
\hline & $\mathrm{D}$ & $\mathrm{P}+\mathrm{O}+$ glucuronide & 635 & 2.04 & $\mathrm{HH}$ \\
\hline \multirow[t]{7}{*}{ Glibenclamide } & & Parent & 494 & 2.76 & $\mathrm{RH}, \mathrm{HH}$ \\
\hline & A & $\mathrm{P}+\mathrm{O}$ & 510 & 2.57 & $\mathrm{RH}, \mathrm{HH}$ \\
\hline & $\mathrm{B}$ & $\mathrm{P}+\mathrm{O}$ & 510 & 2.36 & $\mathrm{RH}, \mathrm{HH}$ \\
\hline & $\mathrm{C}$ & $\mathrm{P}+\mathrm{O}$ & 510 & 2.31 & $\mathrm{RH}, \mathrm{HH}$ \\
\hline & $\mathrm{D}$ & $\mathrm{P}+\mathrm{O}$ & 510 & 2.28 & $\mathrm{RH}, \mathrm{HH}$ \\
\hline & $\mathrm{E}$ & $\mathrm{P}+\mathrm{O}$ & 510 & 2.24 & $\mathrm{RH}, \mathrm{HH}$ \\
\hline & $\mathrm{F}$ & N-dealkylation & 412 & 2.18 & $\mathrm{RH}, \mathrm{HH}$ \\
\hline \multirow{8}{*}{ Troglitazone } & & Parent & 440 & 3.14 & $\mathrm{RH}, \mathrm{HH}$ \\
\hline & A & $\mathrm{P}+\mathrm{O}$ & 456 & 2.89 & $\mathrm{RH}, \mathrm{HH}$ \\
\hline & $\mathrm{B}$ & $\mathrm{P}+$ sulfate & 520 & 2.47 & $\mathrm{RH}, \mathrm{HH}$ \\
\hline & $\mathrm{C}$ & $\mathrm{P}+$ glucuronide & 616 & 2.24 & $\mathrm{RH}, \mathrm{HH}$ \\
\hline & $\mathrm{D}$ & $\mathrm{P}+\mathrm{O}+$ sulfate & 536 & 2.14 & RH \\
\hline & $\mathrm{E}$ & $\mathrm{P}+\mathrm{O}+$ sulfate & 536 & 2.35 & $\mathrm{RH}, \mathrm{HH}$ \\
\hline & $\mathrm{F}$ & $\mathrm{P}+\mathrm{O}+2 \mathrm{H}+$ sulfate & 538 & 2.01 & $\mathrm{RH}, \mathrm{HH}$ \\
\hline & $\mathrm{G}$ & $\mathrm{P}+\mathrm{O}+\mathrm{O}$ & 472 & 2.36 & $\mathrm{RH}, \mathrm{HH}$ \\
\hline \multirow[t]{6}{*}{ Rosiglitazone } & & Parent & 358 & 2.69 & $\mathrm{RH}, \mathrm{HH}$ \\
\hline & A & $\mathrm{P}+\mathrm{O}$ & 374 & 2.27 & $\mathrm{RH}, \mathrm{HH}$ \\
\hline & $\mathrm{B}$ & N-desmethyl & 344 & 2.36 & $\mathrm{RH}, \mathrm{HH}$ \\
\hline & $\mathrm{C}$ & $\mathrm{N}$-desmethyl + O & 360 & 2.00 & $\mathrm{RH}, \mathrm{HH}$ \\
\hline & $\mathrm{D}$ & $\mathrm{P}+\mathrm{O}+$ glucuronide & 550 & 1.92 & $\mathrm{RH}, \mathrm{HH}$ \\
\hline & $\mathrm{E}$ & $\mathrm{P}+\mathrm{O}$ & 374 & 1.97 & $\mathrm{RH}, \mathrm{HH}$ \\
\hline
\end{tabular}

RT, retention time; $\mathrm{RH}$, rat hepatocyte; $\mathrm{HH}$, human hepatocyte.

Cox et al., 2000), it is probable that the expression and/or activity of the CYP2C8 ortholog CYP2C22, a member of rat CYP2C family with homology to human CYP2C8 and CYP2C9 (Qian et al., 2010), is comparable between the two strains. However, in the case of metformin, which is mainly cleared through renal excretion mediated by kidney organic cation transporters (Graham et al., 2011), our findings are not as clear. Therefore, it remains to be determined what differences in expression and/or activity of renal transporters may exist between ZDF and SD rats, and the effects of impaired kidney function on the elimination of drugs, such as metformin, by passive diffusion and transporter-mediated secretion. The differences in PK for other drugs investigated in this study indicated a change in the hepatic metabolism of ZDF rats. Glibenclamide is primarily metabolized in the human liver via P450-mediated oxidative pathways, with CYP3A4, CYP2C9, and CYP2C8 enzymes involved in the formation of multiple oxidative metabolites (Zharikova et al., 2009; Zhou et al., 2010). In rat hepatocytes, the ethylene-hydroxylated glibenclamide (metabolite A) was the most abundant metabolite detected and is formed in human via CYP3A4 (Zharikova et al., 2009), suggesting that the CYP3A4 ortholog in rat is most probably responsible for the hepatic metabolism of glibenclamide.
A
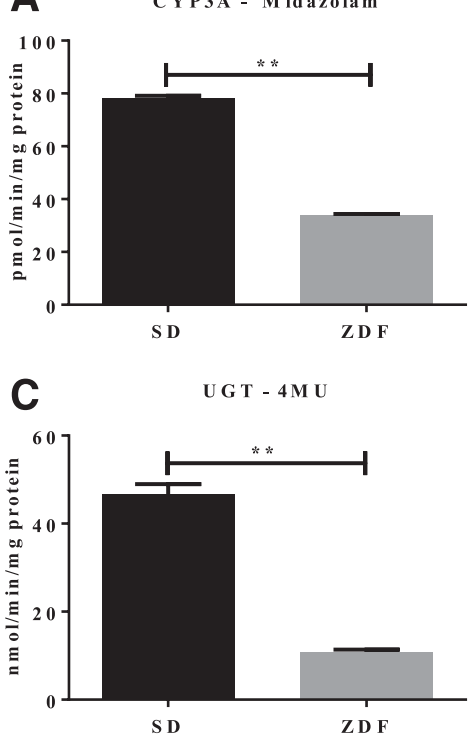

B

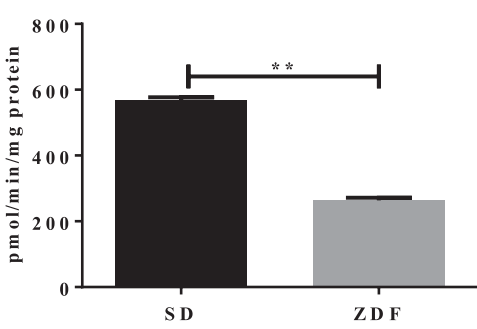

D

SULT - 7-Hydroxycoumarin



Fig. 4. Unbound rate of metabolism of midazolam (A), testosterone (B), 4-methylumbelliferone (4-MU) (C), and 7-hydroxycoumarin (D) by liver $\mathrm{S} 9$ from $\mathrm{SD}$ and $\mathrm{ZDF}$ rats. Formation of $6 \beta$-hydroxytestosterone, 1-hydroxymidazolam, 4-MU glucuronide, and 7-hydroxycoumarin sulfate was determined as described in Materials and Methods. The values presented are mean \pm S.D. $(n=3)$. Statistical analysis was conducted for unbound rate of metabolite formation, ${ }^{* *} P<0.01$ between the two strains. 
A
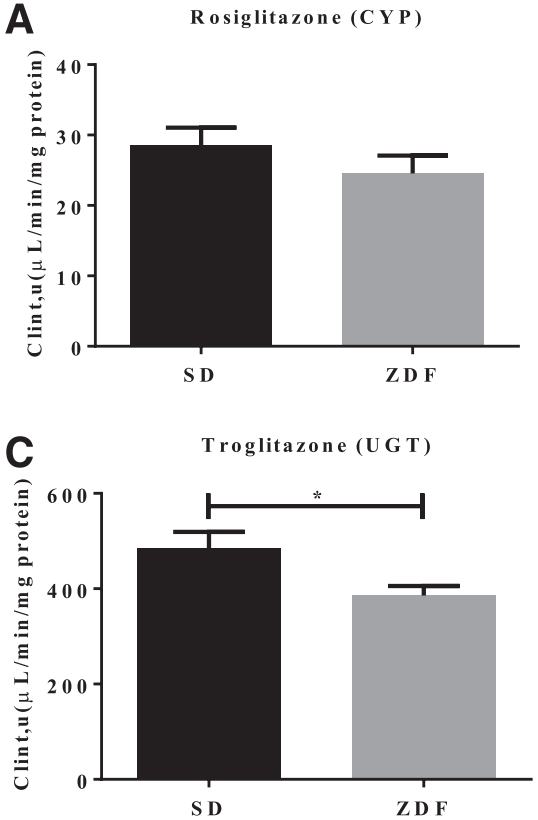

E

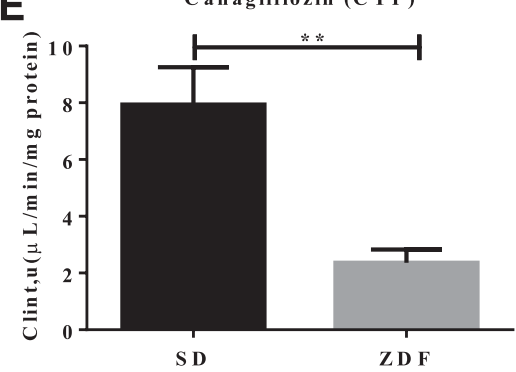

B

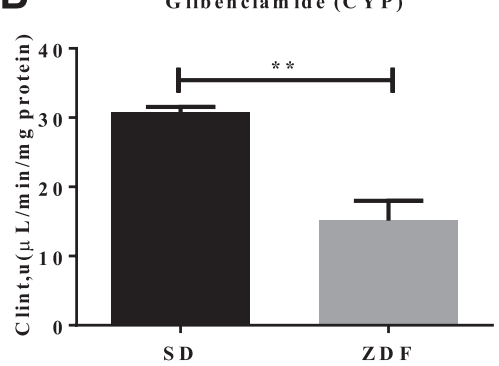

D

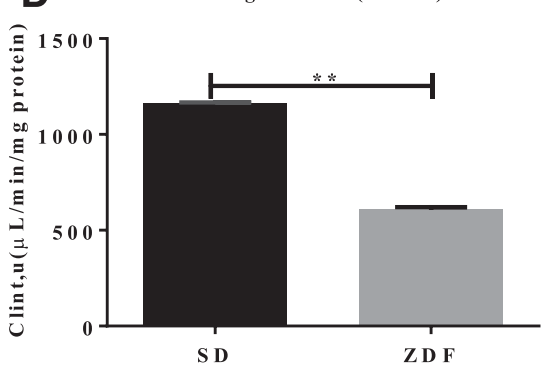

$\mathbf{F}$



Fig. 5. Unbound $\mathrm{CL}_{\text {int }}$ of rosiglitazone (A), glibenclamide (B), troglitazone (C, D), and canagliflozin $(\mathrm{E}, \mathrm{F})$ in liver S9 from SD and ZDF rats. Rate for the parent loss was determined in NAPDH-, UDPGA-, or PAPS-fortified systems as described in Materials and Methods. The values presented are mean \pm S.D. $(n=3)$. Statistical analysis was conducted for unbound $\mathrm{CL}_{\mathrm{int}}, * P<0.05$, **P $P<0.01$ between the two strains.
However, species differences in enzyme substrate specificity have been discovered between rat and human (Martignoni et al., 2006). For example, some human CYP3A phenotypical substrates (e.g., nifedipine) are not metabolized by rat CYP3A1 (Guengerich, 1997). Despite the potential difference, in our current study, the unbound in vitro $C L_{\text {int }}$ for glibenclamide in a NADPH-fortified system was significantly lower in ZDF compared with SD rats, consistent with decreased CYP3A activity toward the typical probe substrates testosterone and midazolam. Therefore, the difference in plasma clearance between the two strains can be explained by a diminished hepatic CYP3A ortholog metabolism in the ZDF rat. Conversely, the results for other compounds were not as direct. Species difference in metabolite formation from canagliflozin was reported in the in vivo metabolite identification experiments. UGTmediated $O$-glucuronidation was the major clearance pathway in human, whereas in rats, oxidation and oxidation-plus-glucuronidation products were the predominant pathways, with the alcohol oxidation of metabolite A being most abundant (Mamidi et al., 2014). In humans, UGT1A9 and UGT2B4 are responsible for the formation of the two $O$-glucuronidation metabolites, respectively (Francke et al., 2015), and CYP3A4 contributes to the formation of a minor hydroxylation

TABLE 3

Fraction unbound of probe substrates and antidiabetic drugs in liver S9 of ZDF and SD rats

Incubation was conducted with $1 \mu \mathrm{M}$ compound and liver S9 protein concentrations as described in Materials and Methods. Results shown represent mean \pm standard deviation of six replicates.

\begin{tabular}{|c|c|c|c|c|c|c|c|c|}
\hline \multirow{2}{*}{ Probe Substrate } & \multicolumn{2}{|c|}{ Testosterone } & \multicolumn{2}{|c|}{ Midazolam } & \multicolumn{2}{|c|}{ 4-MU } & \multicolumn{2}{|c|}{ 7-Hydroxycoumarin } \\
\hline & SD & ZDF & SD & ZDF & SD & $\mathrm{ZDF}$ & SD & ZDF \\
\hline Fraction unbound & $0.78 \pm 0.08$ & $0.88 \pm 0.05^{a}$ & $0.82 \pm 0.03$ & $0.89 \pm 0.04^{b}$ & $0.92 \pm 0.03$ & $0.93 \pm 0.02$ & $1.00^{c}$ & $1.00^{c}$ \\
\hline \multirow{2}{*}{ Drug } & \multicolumn{2}{|c|}{ Rosiglitazone } & \multicolumn{2}{|c|}{ Glibenclamide } & \multicolumn{2}{|c|}{ Canagliflozin } & \multicolumn{2}{|c|}{ Troglitazone } \\
\hline & SD & $\mathrm{ZDF}$ & SD & $\mathrm{ZDF}$ & SD & $\mathrm{ZDF}$ & SD & $\mathrm{ZDF}$ \\
\hline Fraction unbound & $0.81 \pm 0.01$ & $0.89 \pm 0.04$ & $0.73 \pm 0.01$ & $0.77 \pm 0.05$ & $0.49 \pm 0.03$ & $0.65 \pm 0.05^{b}$ & $0.43 \pm 0.03$ & $0.61 \pm 0.06^{b}$ \\
\hline
\end{tabular}


metabolite (Mamidi et al., 2014), which was not detected as one of the major metabolites in human or rat hepatocytes in the present study. In the substrate depletion assay, no difference in UGT-mediated glucuronidation between the two strains was observed despite significantly lower UGT activity in ZDF compared with SD rats when the UGT probe substrate 4-MU was used. It should be noted that 4-MU is a general UGT probe, and human UGT1A1, 1A3, 1A6, 1A7, 1A8, 1A9, 1A10, 2B7, 2B15, and 2B17 all exhibit activity toward this substrate (Uchaipichat et al., 2004). It is possible that the activity/expression of the rat orthologs responsible for 4-MUG formation are decreased in ZDF rats, whereas the orthologs for human UGT1A9 and UGT2B4 are not altered. Differences in the rate of glucuronidation between obese Zucker and SD or lean littermates have been found to be substrate-dependent (Kim et al., 2004). Lower rates for glucuronidation of two PPAR agonists, owing to decreased expression of UGT1A1, 1A6, and 2B1, were observed in obese Zucker rats compared with SD rats (Kim et al., 2004), but rates of glucuronidation of 1-naphthol, estrone, and morphine were comparable between obese Zucker rats and their lean littermates (Chaudhary et al., 1993). It remains to be determined if the decreased activity observed in the NADPH-fortified in vitro system can explain the lower plasma clearance in vivo for canagliflozin, given that both P450 and other enzymes such as alcohol dehydrogenase and aldehyde dehydrogenase can be involved in the alcohol oxidation to carboxylic acid. Lastly, troglitazone has been shown to be mainly cleared via SULT-mediated sulfation, UGT-mediated glucuronidation, and CYP3A4-mediated quinone formation in human (Loi et al., 1999; He et al., 2001), although the quinone metabolite was not detected in rat or human hepatocytes in our study. Lower in vitro unbound $C L_{\text {int }}$ was detected in both the PAPS- and UDPGA-fortified assays for ZDF rats, consistent with decreased plasma clearance in vivo. Although the SULT activity was not different between the two strains when the probe substrate 7-hydroxycoumarin, a general SULT substrate, was used, it is probable that decreased expression and/or activity of rat ortholog of human SULT1A1, the main enzyme for the metabolism of troglitazone (Honma et al., 2002), is responsible for the lower $C L_{\text {int }}$ of troglitazone depletion in $\mathrm{ZDF}$ rats and subsequently lower in vivo clearance.

In addition to differences in hepatic metabolism, other factors could contribute to differences observed in the PK between ZDF and SD rats. Altered plasma protein binding has been reported for propranolol and diazepam that are bound to plasma $\alpha 1$-acid glycoprotein, and phenytoin that is bound to albumin (Benedek et al., 1985), but not for the two PPAR agonists in obese Zucker rats (Kim et al., 2004). In the present study, all four hepatically cleared drugs displayed very low free fraction $(<0.01)$ that could not be readily quantified in SD rat plasma. However, the fraction unbound measured in liver S9 was greater in ZDF compared with SD rats for several probe substrates and antidiabetic drugs. Since the recovery of S9 protein was comparable between the two strains, subcellular lipid content in liver S9 between the two strains may explain this difference. However, the mechanism for the decreased nonspecific binding remains to be identified and is a topic of further study.

Diabetes has been shown to be accompanied by alterations in expression and activity of hepatic drug metabolism enzymes. Reduced activity of human UGT2B7 was observed in diabetic human liver for formation of acyl-glucuronide of mycophenolic acid, whereas activity of UGT1A1 and UGT1A9 for formation of phenol glucuronide metabolite was not altered (Dostalek et al., 2011a). A significant increase in hepatic CYP2E1 activity, assessed through chlorzoxazone disposition, was observed in obese type 2 patients with diabetes and confirmed through increased protein levels through Western blots and greater chlorzoxazone 6-hydroxylation activity in diabetic human liver microsomes (Wang et al., 2003). In contrast, a significant decrease in hepatic CYP3A4 enzymatic activity and protein level has been associated with diabetes (Dostalek et al., 2011b). Several culprits have been hypothesized for the decreased CYP3A4 expression in diabetic liver. These include the effects of proinflammatory cytokines (Morgan, 1997), noncytokine components, and oxidative stress (Dostalek et al., 2011b). Expression of CYP3A4 was found to be downregulated by interleukin-1 $\beta$, IL-6 (Sunman et al., 2004), interferon- $\gamma$ (Donato et al., 1997), and hepatocyte growth factor (Donato et al., 1998) in vitro in human hepatocytes. Experimental and clinical studies also suggest that oxidative stress is involved in the pathogenesis of diabetes (Dostalek et al., 2011b). Thus, downregulation of P450 in patients with diabetes could be a survival mechanism of cells to prevent the deleterious effects of increased formation of reactive oxygen/ nitrogen species produced in the diseased state (Dostalek et al., 2011b). However, as the etiology and pathogenesis of diabetes is complex, it remains unclear whether the alteration in pathophysiology is the cause of or accompanies the down- and/or upregulation of drug-metabolizing enzymes. Furthermore, it remains to be determined what the consequences are on endogenous and xenobiotic metabolism when expression and activity of drug-metabolizing enzymes are altered. Oxidative and metabolic stress have been confirmed through the induction of nitric oxide synthase, as well as the increased expression of CYP2E1, respectively, in ZDF rats compared with their lean littermates (Raza et al., 2015). In the current study, the activity of CYP3A was lower in ZDF rat and is consistent with reports of decreased hepatic CYP3A4 activity in humans with diabetes (Dostalek et al., 2011b). The consistency in the phenotype of oxidative stress and alteration of expression and activity of drugmetabolizing enzymes suggests that ZDF rats can serve as a nonclinical model for investigating the changes in PK and hepatic metabolism in the diseased state in order to understand the connectivity of pathogenesis of diabetes and alteration in drug metabolism (Raza et al., 2015).

In conclusion, we demonstrate that metabolic capability for some of the drug-metabolizing enzymes in the liver of ZDF rats is decreased compared with SD rats. The discrepancy should raise attention to interpretation of the PK/PD relationship in drug discovery when $\mathrm{PK}$ is conducted in SD and PD is performed in ZDF rats. Future work to understand the mechanism by which the changes in drug-metabolizing enzymes occur in the disease model will help elucidate the PK/PD relationship in the diseased population.

\section{Acknowledgments}

We are grateful to Dr. Stephen Hall and Dr. Daniel Mudra for guidance and helpful discussions. We also thank Dr. Thomas Raub and Dr. Mary Pat Knadler for comments on the manuscript.

\section{Authorship Contributions}

Participated in research design: Zhou, Rougée, Cramer, Mohutsky, Yumibe, Adams.

Conducted experiments: Rougée, Bedwell.

Contributed new reagents or analytic tools: Bedwell, Ruterbories, Moulton, Mohutsky.

Performed data analysis: Zhou, Rougée, Calvert, Cassidy.

Wrote or contributed to the writing of the manuscript: Zhou, Rougée, Cassidy, Bedwell, Mohutsky.

\section{References}

Aleixandre de Artiñano A and Miguel Castro M (2009) Experimental rat models to study the metabolic syndrome. Br J Nutr 102:1246-1253.

Baldwin SJ, Clarke SE, and Chenery RJ (1999) Characterization of the cytochrome P450 enzymes involved in the in vitro metabolism of rosiglitazone. Br J Clin Pharmacol 48:424-432.

Benedek IH, Blouin RA, and McNamara PJ (1985) Altered drug-serum protein binding in the genetically obese Zucker rat. J Pharm Sci 74:837-840.

Bhattia SK, Hadden DR, Montgomery DA, and Weaver JA (1970) Glibenclamide therapy in diabetes mellitus. BMJ 2:570-572.

Brouwer KLR, Kostenbauder HB, McNamara PJ, and Blouin RA (1984) Phenobarbital in the genetically obese Zucker rat. I. Pharmacokinetics after acute and chronic administration. $J$ Pharmacol Exp Ther 231:649-653. 
Chaudhary IP, Tuntaterdtum S, McNamara PJ, Robertson LW, and Blouin RA (1993) Effect of genetic obesity and phenobarbital treatment on the hepatic conjugation pathways. J Pharmacol Exp Ther 265: $1333-1338$.

Clark JB, Palmer CJ, and Shaw WN (1983) The diabetic Zucker fatty rat. Proc Soc Exp Biol Med 173:68-75.

Cox PJ, Ryan DA, Hollis FJ, Harris AM, Miller AK, Vousden M, and Cowley H (2000) Absorption, disposition, and metabolism of rosiglitazone, a potent thiazolidinedione insulin sensitizer, in humans. Drug Metab Dispos 28:772-780.

Davies B and Morris T (1993) Physiological parameters in laboratory animals and humans. Pharm Res 10:1093-1095.

Donato MT, Gómez-Lechón MJ, Jover R, Nakamura T, and Castell JV (1998) Human hepatocyte growth factor down-regulates the expression of cytochrome P450 isozymes in human hepatocytes in primary culture. J Pharmacol Exp Ther 284:760-767.

Donato MT, Guillén MI, Jover R, Castell JV, and Gómez-Lechón MJ (1997) Nitric oxide-mediated inhibition of cytochrome $\mathrm{P} 450$ by interferon-gamma in human hepatocytes. J Pharmacol Exp Ther 281:484-490.

Dostalek M, Court MH, Hazarika S, and Akhlaghi F (2011a) Diabetes mellitus reduces activity of human UDP-glucuronosyltransferase 2B7 in liver and kidney leading to decreased formation of mycophenolic acid acyl-glucuronide metabolite. Drug Metab Dispos 39:448-455.

Dostalek M, Court MH, Yan B, and Akhlaghi F (2011b) Significantly reduced cytochrome P450 $3 \mathrm{~A} 4$ expression and activity in liver from humans with diabetes mellitus. $\mathrm{Br} J$ Pharmacol 163: 937-947.

Enriquez A, Leclercq I, Farrell GC, and Robertson G (1999) Altered expression of hepatic CYP2E1 and CYP4A in obese, diabetic ob/ob mice, and fa/fa Zucker rats. Biochem Biophys Res Commun 255:300-306.

Francke S, Mamidi RN, Solanki B, Scheers E, Jadwin A, Favis R, and Devineni D (2015) In vitro metabolism of canagliflozin in human liver, kidney, intestine microsomes, and recombinant uridine diphosphate glucuronosyltransferases (UGT) and the effect of genetic variability of UGT enzymes on the pharmacokinetics of canagliflozin in humans. J Clin Pharmacol 55:1061-1072.

Fujiwara T, Yoshioka S, Yoshioka T, Ushiyama I, and Horikoshi H (1988) Characterization of new oral antidiabetic agent CS-045. Studies in KK and ob/ob mice and Zucker fatty rats. Diabetes 37 $1549-1558$.

Giroux M-C, Santamaria R, Hélie P, Burns P, Beaudry F, and Vachon P (2016) Physiological, pharmacokinetic and liver metabolism comparisons between 3-, 6-, 12- and 18-month-old male Sprague Dawley rats under ketamine-xylazine anesthesia. Exp Anim 65:63-75.

Graham GG, Punt J, Arora M, Day RO, Doogue MP, Duong JK, Furlong TJ, Greenfield JR, Greenup LC, and Kirkpatrick CM, et al. (2011) Clinical pharmacokinetics of metformin. Clin Pharmacokinet 50:81-98.

Guengerich FP (1997) Comparisons of catalytic selectivity of cytochrome P450 subfamily enzymes from different species. Chem Biol Interact 106:161-182.

He K, Woolf TF, Kindt EK, Fielder AE, and Talaat RE (2001) Troglitazone quinone formation catalyzed by human and rat CYP3A: an atypical CYP oxidation reaction. Biochem Pharmacol 62:191-198.

Honma W, Shimada M, Sasano H, Ozawa S, Miyata M, Nagata K, Ikeda T, and Yamazoe Y (2002) Phenol sulfotransferase, ST1A3, as the main enzyme catalyzing sulfation of troglitazone in human liver. Drug Metab Dispos 30:944-949.

Kava R, Greenwood MRC, and Johnson PR (1990) New rat models of obesity and type II diabetes: Zucker ( $f a / f a)$ Rat. ILAR J 32:4-88.

Kim MS, Wang S, Shen Z, Kochansky CJ, Strauss JR, Franklin RB, and Vincent SH (2004) Differences in the pharmacokinetics of peroxisome proliferator-activated receptor agonists in genetically obese Zucker and sprague-dawley rats: implications of decreased glucuronidation in obese Zucker rats. Drug Metab Dispos 32:909-914.

Lehmann JM, Moore LB, Smith-Oliver TA, Wilkison WO, Willson TM, and Kliewer SA (1995) An antidiabetic thiazolidinedione is a high affinity ligand for peroxisome proliferator-activated receptor gamma (PPAR gamma). J Biol Chem 270:12953-12956.

Litterst CL (1980) In vitro hepatic drug metabolism and microsomal enzyme induction in genetically obese rats. Biochem Pharmacol 29:289-296.

Loi CM, Young M, Randinitis E, Vassos A, and Koup JR (1999) Clinical pharmacokinetics of troglitazone. Clin Pharmacokinet 37:91-104.
Mamidi RN, Cuyckens F, Chen J, Scheers E, Kalamaridis D, Lin R, Silva J, Sha S, Evans DC, and Kelley MF, et al. (2014) Metabolism and excretion of canagliflozin in mice, rats, dogs, and humans. Drug Metab Dispos 42:903-916.

Martignoni M, Groothuis GM, and de Kanter R (2006) Species differences between mouse, rat, dog, monkey and human CYP-mediated drug metabolism, inhibition and induction. Expert Opin Drug Metab Toxicol 2:875-894.

Morgan ET (1997) Regulation of cytochromes P450 during inflammation and infection. Drug Metab Rev 29:1129-1188

Ning M, Li L, Li J, Li Z, Li R, Zhou T, and Lu W (2012) In vitro screening of reversible and timedependent inhibition on CYP3A by TM208 and TM209 in rat liver microsomes. Acta Pharm Sin B 2:181-187.

Nomura S, Sakamaki S, Hongu M, Kawanishi E, Koga Y, Sakamoto T, Yamamoto Y, Ueta K, Kimata H, and Nakayama K, et al. (2010) Discovery of canagliflozin, a novel C-glucoside with thiophene ring, as sodium-dependent glucose cotransporter 2 inhibitor for the treatment of type 2 diabetes mellitus. $J$ Med Chem 53:6355-6360.

Peterson RG, Shaw WN, Neel MA, Little LA, and Eichberg J (1990) Zucker diabetic fatty rat as a model for non-insulin-dependent diabetes mellitus. ILAR J 32:16-19.

Phillips MS, Liu Q, Hammond HA, Dugan V, Hey PJ, Caskey CJ, and Hess JF (1996) Leptin receptor missense mutation in the fatty Zucker rat. Nat Genet 13:18-19.

Qian L, Zolfaghari R, and Ross AC (2010) Liver-specific cytochrome P450 CYP2C22 is a direct target of retinoic acid and a retinoic acid-metabolizing enzyme in rat liver. J Lipid Res 51: $1781-1792$.

Raza H, John A, and Howarth FC (2015) Increased oxidative stress and mitochondrial dysfunction in zucker diabetic rat liver and brain. Cell Physiol Biochem 35:1241-1251.

Shiota M and Printz RL (2012) Diabetes in Zucker diabetic fatty rat. Methods Mol Biol 933: $103-123$.

Shiratani H, Katoh M, Nakajima M, and Yokoi T (2008) Species differences in UDPglucuronosyltransferase activities in mice and rats. Drug Metab Dispos 36:1745-1752.

Sterne J (1959) [Treatment of diabetes mellitus with N,N-dimethylguanylguanidine (LA. 6023, glucophage)]. Therapie 14:625-630.

Sunman JA, Hawke RL, LeCluyse EL, and Kashuba AD (2004) Kupffer cell-mediated IL-2 suppression of CYP3A activity in human hepatocytes. Drug Metab Dispos 32:359-363.

Truett GE, Bahary N, Friedman JM, and Leibel RL (1991) Rat obesity gene fatty (fa) maps to chromosome 5: evidence for homology with the mouse gene diabetes (db). Proc Natl Acad Sci USA 88:7806-7809.

Uchaipichat V, Mackenzie PI, Guo XH, Gardner-Stephen D, Galetin A, Houston JB, and Miners JO (2004) Human udp-glucuronosyltransferases: isoform selectivity and kinetics of 4-methylumbelliferone and 1-naphthol glucuronidation, effects of organic solvents, and inhibition by diclofenac and probenecid. Drug Metab Dispos 32:413-423.

van den Berg SJ, Klaus V, Alhusainy W, and Rietjens IM (2013) Matrix-derived combination effect and risk assessment for estragole from basil-containing plant food supplements (PFS). Food Chem Toxicol 62:32-40.

Wang Z, Hall SD, Maya JF, Li L, Asghar A, and Gorski JC (2003) Diabetes mellitus increases the in vivo activity of cytochrome P450 2E1 in humans. Br J Clin Pharmacol 55:77-85.

Zamek-Gliszczynski MJ, Ruterbories KJ, Ajamie RT, Wickremsinhe ER, Pothuri L, Rao MV, Basavanakatti VN, Pinjari J, Ramanathan VK, and Chaudhary AK (2011) Validation of 96-wel equilibrium dialysis with non-radiolabeled drug for definitive measurement of protein binding and application to clinical development of highly-bound drugs. J Pharm Sci 100:2498-2507.

Zharikova OL, Fokina VM, Nanovskaya TN, Hill RA, Mattison DR, Hankins GD, and Ahmed MS (2009) Identification of the major human hepatic and placental enzymes responsible for the biotransformation of glyburide. Biochem Pharmacol 78:1483-1490.

Zhou L, Naraharisetti SB, Liu L, Wang H, Lin YS, Isoherranen N, Unadkat JD, Hebert MF, and Mao Q (2010) Contributions of human cytochrome P450 enzymes to glyburide metabolism. Biopharm Drug Dispos 31:228-242.

Address correspondence to: Dr. Xin Zhou, Eli Lilly and Company, Corporate Center, Drop Code 0710, Indianapolis, IN 46285. E-mail: zhou_xin@lilly.com 